\title{
Late postoperative slippage of the cerebral aneurysm clip. A systematic review and meta-analysis
}

\author{
Tomasz Szmuda $\odot$, Paweł Słoniewski
}

Department of Neurosurgery, Medical University of Gdańsk, Poland

\begin{abstract}
Background: A late clip slippage from the previously properly secured cerebral aneurysm is rarely observed. To date these complications have not pooled and evaluated using systematic review methodology. The objective was to report factors attributed to the late slippage of the aneurysm clip in the postoperative period. Materials and methods: All causes of postoperative clip slippage were systematically reviewed and analysed according to PRISMA Individual Patient Data protocol. Medline (PubMed), Embase, Cochrane, ISI Web of Knowledge and Google Scholar were searched for all relevant cases. Results: Systematic review of the literature yielded 105 original cases proving slipped clip in the postoperative period. The slipped clip caused bleeding in $53.8 \%$ of patients. The putative cause of clip slippage was provided in only $34.7 \%$ of the published cases. If a single clip was used, then complete clip slippage was noted more often $(p=0.04)$. Multiple clipping and clip-wrapping techniques were postulated as ways to prevent postoperative clip slippage. Conclusions: The reason for late slippage of the aneurysm clip remains unexplained by most authors. Based on systematic reviewing, the use of tandem of clips prevents their late migration off the aneurysm. Clipping with wrapping or use of a single clip reinforced by any wrapping material seems a more durable solution.

Keywords: systematic review • intracranial aneurysm • clip slippage • neurosurgical clipping
\end{abstract}

\section{Citation}

Szmuda T, Słoniewski P. Late postoperative slippage of the cerebral aneurysm clip. A systematic review and meta-analysis. Eur J Transl Clin Med. 2019;2(1):56-69.

DOI: $10.31373 /$ ejtcm/103442

Corresponding author:

Tomasz Szmuda, Department of Neurosurgery, Medical University of Gdańsk, Poland

e-mail: tszmuda@gumed.edu.pl

No external funds.

Available online: www.ejtcm.gumed.edu.pl

Copyright ${ }^{\circledR}$ Medical University of Gdańsk

This is Open Access article distributed under the terms of the Creative Commons Attribution-ShareAlike 4.0 International. 


\section{Background}

Postoperative clip slippage is a rarely observed complication. Authors attributed this complication to the application of a short clip, some alloy features or clip closing pressure $[1,2]$. Repetitive opening of the clip further reduces its closing forces [3-5]. Another factor is the so-called scissoring effect [6-9]. Various authors demonstrated the examples of late clip migration following seemingly successful operations [10-13]. Less than $1 \%$ of the postoperative angiograms show an insufficiently secured aneurysm or a rotated clip [14]. Authors demonstrated various techniques in order to avoid clip slippage, although their interests were focused on the particular operative environment. Hundreds of case reports that were never critically appraised. To date, cases of clip slippage have not been pooled and analysed using a validated systematic review methodology. We aimed to collect and summarize the existing literature about clip slippage phenomena using the reproducible and widely accepted PRISMA Statement methodology [15].

\section{Methods and materials}

After reaching consensus, the authors developed a detailed protocol [16]. One author searched (November 2017), selected the articles and extracted data. Online Medline, Embase, Web of Knowledge, Cochrane and Google Scholar engines were queried for phrase: 'aneurysm' AND ('clip' OR 'clipping') AND ('slip' OR 'slippage'). Duplicating records were removed using Mendeley Software (ver.1.17.10). Screening was based on titles and abstracts. We accepted original case reports, reviews, commentaries, expert opinions including animal, technical studies, $\mathrm{PhD}$ dissertations and patents. No limits in time of publication or language were applied. Google Translate website was used in case of abstracts and articles not in English. Following eligibility assessment, extensive searches for relevant references followed data extraction. Two types of data were deemed valid for further narrative synthesis of evidence: (1) descriptions of postoperative clip slippage and (2) intraoperative manoeuvres intended for prevention of a late slip-off phenomena. Raw data from each patient (Individual Participant Data method, IPD) were analysed as if all slippage occurrences belonged to an assumed single cohort. The evaluation was performed in accordance to Preferred Reporting Items for Systematic Reviews and Meta-Analyses (PRISMA) statement for IPD systematic reviews, which included search, eligibility, extraction, and reporting [17]. Publication bias was not assessed.

We used typical statistical methods for relevant comparisons: chi-square, t-test or Mann-Whitney $U$ test. Probability value less than 0.05 was considered significant. Statistica v. 13.1 (StatSoft Co, Tulsa, OK; USA) and Prism (GraphPad Software, La Jolla, CA; USA) were used. IRB Committee in the institution of systematic reviews is exempt.

\section{Results}

The literature search yielded 3034 records, mostly identified via Google Scholar which explores full-texts for keywords. 'Slip' unrelated to cerebral aneurysm was the main exclusion criterion. Finally, 139 studies were included for the synthesis.

We found 105 original cases reporting late clip migration. In a half of the cases the slipped clip caused bleeding (53.8\%; 43/80), half of which were fatal (23/43). A routine postoperative angiography revealed the incidental clip displacement in $32.5 \%$ of cases (26/80). Anterior communicating artery $(n=15)$, internal carotid artery ( $n=21$, including 2 blister-like), middle cerebral artery $(n=12)$ and basilar artery $(n=7)$ were commonly encountered locations. In majority of cases $(65.3 \%$; $62 / 95)$ the authors were not able to provide any reason for clip slippage. Others blamed the defect of clip material in 15 patients (16.0\%) and persistent arterial pulsation in 6 (6.4\%). Surprisingly, specific features of the particular aneurysm were attributed to only 5 cases of slipped clips (5.3\%).

The published reports rarely included aneurysm size, usually only if the aneurysm was giant $(85.0 \%$; $8 / 10)$. In a quarter of the slippage cases more than one clip was applied (23.3\%; 10/43). In $82.9 \%$ of cases $(68 / 80)$ a clip completely slipped off the aneurysm dome. Aneurysm location $(p=0.65)$, size $(p=0.26)$, rebleeding as the first symptom $(p=0.65)$, fatal rebleeding $(p=0.89)$ and occurrence in postoperative DSA $(p=0.52)$ were not related to the degree (complete or incomplete) of clip migration. On the other hand, complete clip slip-off was significantly more often encountered if a single clip was used $(82.1 \%$ vs. $44.4 \%$, $p=0.04)$. Regarding the direction of slip, in $87.5 \%$ of reports the clip migrated off the aneurysm $(87.5 \%$; $70 / 80)$, whereas in 8 cases $(11.4 \%)$ the clip was displaced down onto the parent vessel, causing cerebral ischemia in 3 patents (37.5\%).

Out of the 139 studies, 78 (56.1\%) provided at least one suggestion on how to avoid late clip migration. The most commonly suggested method was placing several clips instead of one (15.8\%; 24/139), followed by applying of clip-wrapping technique (7.2\%p; $10 / 139)$ and performing DSA shortly after clipping (4.3\%; 6/139). The suggested preventive methods were location-specific, e.g. in case of blood blister-like aneurysm authors postulated placing clips parallel to ICA or clip-wrapping. 

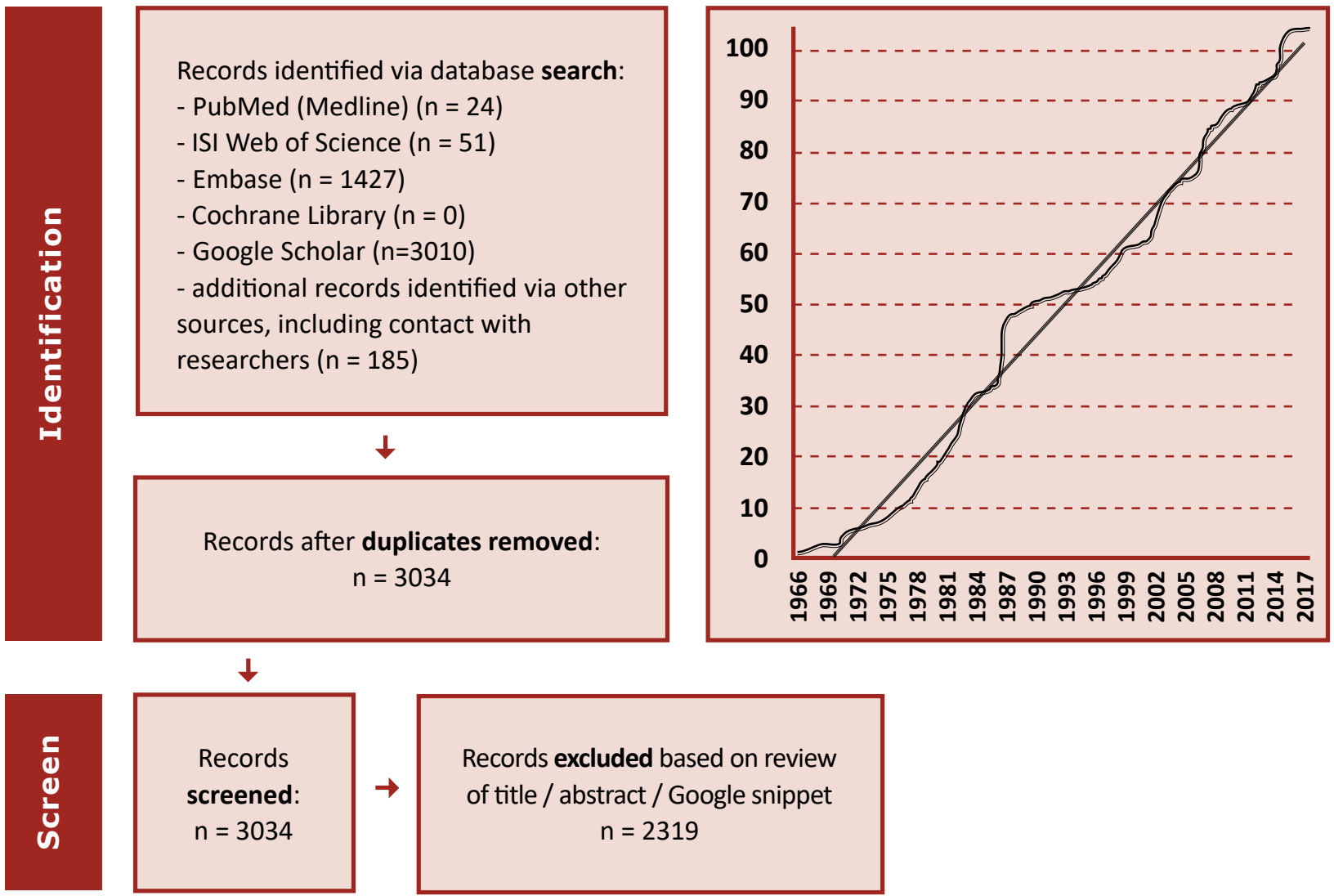

Records excluded based on review of title / abstract / Google snippet $\mathrm{n}=2319$

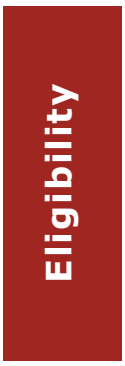

$\downarrow$

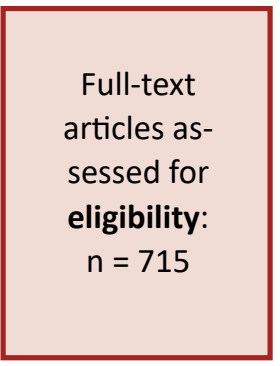

Reasons for full-text article exclusion ( $n=576)$ :

- intraoperative slippage only ( $n=34)$

- alloy features, mechanical testing, not followed by

the examination within clinical cases $(n=62)$

- only alluded about the possibility of slippage ( $n=96)$.

- clip behavior under MRI ( $n=16)$

- slip-off did not occur in the series $(n=19)$

- cited another report/repeated others' conclusion ( $n=42)$

$\downarrow$
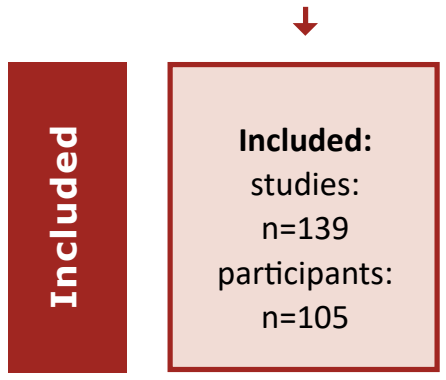

- extracranial aneurysm clip slippage $(n=18)$

- the term 'slip' unrelated to the aneurysm clip ( $n=202)$

- the term 'slip' not mentioned at all ( $n=19)$

- insufficient data, full-text file not available

or translation not understandable $(n=18)$

- duplicated search record or series $(n=49)$

Figure 1. Flowchart depicting the strategy for literature search. Cumulative number of cases involving aneurysm clip slippage.

The graph demonstrates the constantly increasing publication rate on this subject.

From: Moher D, Liberati A, Tetzlaff J, Altman DG, The PRISMA Group (2009). Preferred Reporting Items for Systematic

Reviews and Meta-Analyses: The PRISMA Statement. PLoS Med 6(7): e1000097. 
Supplementary Table 1. Full table of studies included in the evidence synthesis.
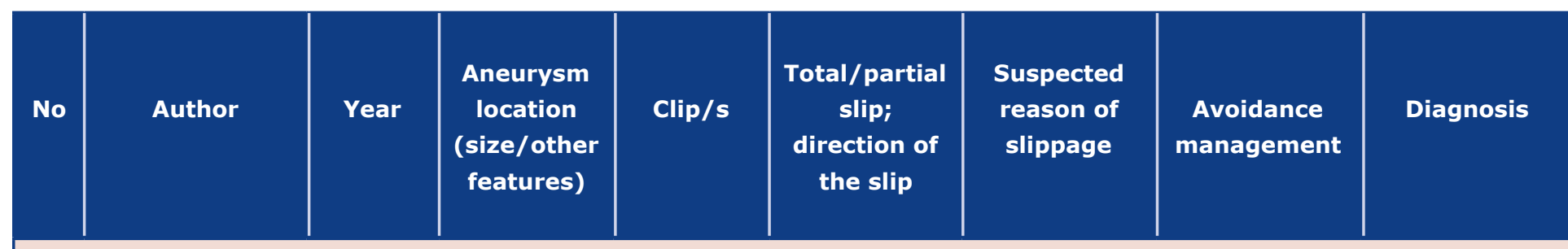

\section{MCA}

\begin{tabular}{|c|c|c|c|c|c|c|c|c|}
\hline 1 & Matsumoto [1] & 1987 & MCA & unk & in & \multirow{3}{*}{\multicolumn{2}{|c|}{$\begin{array}{c}\text { clip properties; } \\
\text { authors presented } \\
\text { a modified non-sliding clip, } \\
\text { which blades closed } \\
\text { at their tips. }\end{array}$}} & MCA obstruction \\
\hline 2 & Matsumoto [1] & 1987 & MCA & Yasargil & total; out & & & postop DSA \\
\hline 3 & Matsumoto [1] & 1987 & MCA & unk & partial; out & & & oculomotor paresis \\
\hline 5 & Nakayama [3] & 1987 & MCA & $\begin{array}{l}\text { misused } \\
\text { temporary } \\
\text { clip }\end{array}$ & total; out & unk & none & $\begin{array}{l}\text { rebleeding } \\
(1.5 \text { mths })\end{array}$ \\
\hline 7 & Hoh [5] & 2001 & MCA & unk & total; out & unk & none & $\begin{array}{c}\text { rebleeding after } \\
3 \text { weeks }\end{array}$ \\
\hline 8 & Asgari [6] & 2003 & MCA & 1 Sugita & partial; out & unk & none & $\begin{array}{l}\text { clip slippage } \\
\text { not verified }\end{array}$ \\
\hline 9 & Wester [7] & 2009 & $\begin{array}{c}\text { MCA } \\
\text { bifurcation }\end{array}$ & curved & total; out & $\begin{array}{l}\text { low closing } \\
\text { forces of the } \\
\text { long clip }\end{array}$ & $\begin{array}{l}\text { Instead of } \\
\text { one long clip, } \\
\text { multiple short } \\
\text { clips should be } \\
\text { used to } \\
\text { reconstruct } \\
\text { the artery. }\end{array}$ & $\begin{array}{l}\text { fatal rebleeding } \\
\text { (after closure } \\
\text { of the wound) }\end{array}$ \\
\hline 11 & Takahashi [8] & 1987 & giant MCA & Sugita & total; out & & none & rebleeding (4d) \\
\hline 12 & Asgari [6] & 2003 & giant MCA & 2 Sugita & partial; out & $\begin{array}{l}\text { wide calcified } \\
\text { neck; only } \\
\text { distal } 2 / 3 \text { of } \\
\text { clip grasped } \\
\text { the neck }\end{array}$ & none & rebleeding \\
\hline 13 & Pia [9] & 1980 & giant MCA & 2 clips & total; out & unk & none & unk \\
\hline
\end{tabular}




\begin{tabular}{|c|c|c|c|c|c|c|c|c|}
\hline \multicolumn{9}{|c|}{ ACOA } \\
\hline 14 & Kandel [10] & 1977 & ACoA & unk & total; out & unk & none & unk \\
\hline 15 & Czochra [11] & 1980 & ACoA & unk & total; out & unk & none & postop DSA \\
\hline 16 & Sakurai [12] & 1987 & $\mathrm{ACoA}$ & $\begin{array}{l}\text { clip and } \\
\text { wrapping }\end{array}$ & total; out & unk & none & $\begin{array}{l}\text { rebleeding } \\
\text { ( } 3 \text { mths) }\end{array}$ \\
\hline 17 & Haraoka [13] & 1987 & $\mathrm{ACoA}$ & $\begin{array}{l}\text { encom- } \\
\text { passing } \\
\text { Heifetz }\end{array}$ & total; out & $\begin{array}{l}\text { incompletely } \\
\text { obliterated } \\
\text { neck and } \\
\text { pulsative } \\
\text { forces to the } \\
\text { neck over } \\
\text { a long period }\end{array}$ & none & good recovery \\
\hline 18 & Asgari [6] & 2003 & $\begin{array}{c}\text { medium } \\
\text {-sized } A C o A\end{array}$ & $\begin{array}{l}1 \text { Yasargil Ti } \\
\text { (Aesculap) }\end{array}$ & partial; out & $\begin{array}{l}\text { should be } \\
\text { differentiated } \\
\text { with de novo } \\
\text { aneurysm }\end{array}$ & none & rebleeding \\
\hline 19 & Fukui [14] & 2004 & ACoA & unk & unk; out & unk & none & unk \\
\hline 20 & Hayashi [15] & 2004 & $\mathrm{ACOA}$ & straight & total; out & $\begin{array}{l}\text { clip head trap- } \\
\text { ped between } \\
\text { optic nerves }\end{array}$ & neurosurgeons & rebleeding $(4 \mathrm{~d})$ \\
\hline 21 & Chen [16] & 2009 & $\mathrm{ACoA}$ & 1 titanium & total; out & unk & none & rebleeding \\
\hline 22 & Huh [17] & 2012 & $\mathrm{ACoA}$ & $\begin{array}{l}\text { single clip } \\
\text { reinforced } \\
\text { by a } \\
\text { booster clip }\end{array}$ & total; out & unk & none & rebleeding \\
\hline 23 & Kunert [18] & 2012 & ACoA & unk & total; out & unexplained & none & control CTA \\
\hline 24 & Takahashi [8] & 1987 & $\mathrm{ACOA}$ & $\begin{array}{l}\text { clipping+- } \\
\text { cyanoacry- } \\
\text { late glue }\end{array}$ & total; out & $\begin{array}{c}\text { selection } \\
\text { of an } \\
\text { inappropriate } \\
\text { clip, inaccurate }\end{array}$ & none & $\begin{array}{l}\text { fatal rebleeding } \\
\qquad(11 \mathrm{~d})\end{array}$ \\
\hline 25 & Takahashi [8] & 1987 & ACoA & unk & total; out & placement & none & rebleeding (17 d) \\
\hline 26 & Yi [19] & 2003 & $\mathrm{ACOA}$ & $\begin{array}{l}\text { bayonet } \\
\text { standard } \\
\text { Yasargil } \\
\text { (Aesculap) }\end{array}$ & total; out & $\begin{array}{l}2 \mathrm{~mm} \text { of neck } \\
\text { remnant was } \\
\text { supposed }\end{array}$ & none & postop DSA \\
\hline 27 & Xuejian [20] & 1998 & ACoA & unk & total out & unk & none & fatal rebleeding \\
\hline 28 & Yasui [21] & 2004 & giant $\mathrm{ACOA}$ & unk & in & $\begin{array}{l}\text { the aneurysm } \\
\text { was approached } \\
\text { from interhemis- } \\
\text { pheric approach }\end{array}$ & none & $\begin{array}{c}\text { occlusion } \\
\text { of parent } \\
\text { artery; } \\
\text { infarction }\end{array}$ \\
\hline
\end{tabular}




\begin{tabular}{|c|c|c|c|c|c|c|c|c|}
\hline 29 & Izumo [22] & 2013 & $\mathrm{~A} 1$ & curved Ti & partial; out & unk & none & postop DSA \\
\hline 30 & Iida [23] & 2017 & fusiform A1 & straight & total; out & unk & none & rebleeding \\
\hline
\end{tabular}

ICA

\begin{tabular}{|c|c|c|c|c|c|c|c|c|}
\hline 31 & Skultety [24] & 1966 & ICA & unk & unk & unk & none & fatal \\
\hline 32 & Sato [25] & 1971 & ICA & long, silver & total; out & $\begin{array}{l}\text { presumably } \\
\text { due to arterial } \\
\text { pulsations }\end{array}$ & none & $\begin{array}{l}\text { uneventful } \\
\text { clinical } \\
\text { course }\end{array}$ \\
\hline 33 & Kariyattil [26] & 2013 & ICA & $\begin{array}{l}\text { bayonet- } \\
\text {-shaped } \\
\text { fenestrated } \\
\text { Yasargil }\end{array}$ & partial; out & \multicolumn{3}{|c|}{$\begin{array}{l}\text { Intraop DSA is advised as revealed clip } \\
\text { "scissoring effect" causing slippage } \\
\text { after apparent right clipping. }\end{array}$} \\
\hline 34 & Edner [2] & 1978 & ICA/PCoA & $\begin{array}{l}\text { straight } \\
\text { Heifetz }\end{array}$ & total; out & $\begin{array}{l}\text { clip head trap- } \\
\text { ped between } \\
\text { optic nerves }\end{array}$ & neurosurgeons & rebleeding $(4 \mathrm{~d})$ \\
\hline 35 & Sengupta [27] & 1978 & ICA/PCoA & 1 unk & total; out & unk & none & fatal rebleeding \\
\hline 36 & Czochra [11] & 1980 & ICA/PCoA & unk & total; out & unk & none & postop DSA \\
\hline 37 & Ebina [28] & 1982 & ICA/PCoA & $\begin{array}{l}\text { Heifetz, } \\
\text { then } \\
\text { Sugita }\end{array}$ & total; out & unk & none & rebleeding \\
\hline 38 & Horiuchi [29] & 2012 & ICA/PCoA & $\begin{array}{l}\text { Yasargil } \\
\text { titanium } \\
\text { bayonet }\end{array}$ & in & $\begin{array}{l}\text { scissoring } \\
\text { effect }\end{array}$ & $\begin{array}{l}\text { remove } \\
\text { immediately } \\
\text { scissor-like } \\
\text { deformed clip }\end{array}$ & $\begin{array}{c}\text { arterial } \\
\text { occlusion (paresis) }\end{array}$ \\
\hline 39 & Drake [30] & 1973 & $\begin{array}{l}\text { board- } \\
\text {-based } \\
\text { ICA/PCoA }\end{array}$ & 1 Sundt & total; out & $\begin{array}{l}\text { improper } \\
\text { clipping; } \\
\text { postoperative } \\
\text { hypertension? }\end{array}$ & $\begin{array}{l}\text { intraop and } \\
\text { postop DSA; } \\
\text { clipping under } \\
\text { deep hypoten- } \\
\text { sion; clip sho- } \\
\text { uld be fenestra- } \\
\text { ted or occludes } \\
\text { partially the } \\
\text { arterial lumen. }\end{array}$ & $\begin{array}{c}\text { clip } \\
\text { slipped } \\
\text { two times }\end{array}$ \\
\hline 40 & Ikezaki [31] & 1987 & 2 ICA/Opth & $\begin{array}{l}\text { tandem } \\
\text { of angle } \\
\text { fenestrated }\end{array}$ & partial; in & unk & $\begin{array}{l}\text { The blades } \\
\text { should be } \\
\text { applied parallel } \\
\text { to ICA lumen }\end{array}$ & $\begin{array}{c}\text { ICA } \\
\text { stenosis }\end{array}$ \\
\hline 41 & Drake [32] & 1984 & ICA/Opth & 1 Sundt & total; out & unk & postop DSA & rebleeding \\
\hline 42 & $\begin{array}{c}\text { Hatanaka } \\
{[33,34]}\end{array}$ & 1987 & ICA/Opth & unk & total; out & unk & $\begin{array}{l}\text { glue applied on } \\
\text { the clip spring }\end{array}$ & rebleeding \\
\hline
\end{tabular}




\begin{tabular}{|c|c|c|c|c|c|c|c|c|}
\hline 43 & Melo [35] & 2002 & $\begin{array}{l}\text { giant } \\
\text { ICA/ } \\
\text { Opth }\end{array}$ & unk & total; out & $\begin{array}{l}\text { weak clip } \\
\text { closing } \\
\text { pressure }\end{array}$ & $\begin{array}{l}\text { do not } \\
\text { resterilize clips; } \\
\text { repeat other } \\
\text { suggestions } \\
\text { to prevent } \\
\text { slipping }\end{array}$ & $\begin{array}{c}\text { postop } \\
\text { DSA } \\
\text { (8 mths) }\end{array}$ \\
\hline 45 & Nemoto [37] & 1999 & paraclinoid ICA & 2 clips & total; out & unk & none & postop DSA \\
\hline 46 & Heros [38] & 1983 & $\begin{array}{c}\text { giant } \\
\text { paraclinoid } \\
\text { ICA }\end{array}$ & unk & total; out & $\begin{array}{l}\text { the reinforcing } \\
\text { clip blades } \\
\text { ruptured the } \\
\text { sac while } \\
\text { slipping }\end{array}$ & $\begin{array}{l}\text { partial neck } \\
\text { clipping with } \\
\text { single clip even } \\
\text { reinforced by } \\
\text { another one } \\
\text { should be } \\
\text { avoided. }\end{array}$ & fatal rebleeding \\
\hline 47 & Szmuda [39] & 2012 & giant ICA & $\begin{array}{c}2 \text { straight, } \\
1 \text { bayonet } \\
\text { Yasargil. }\end{array}$ & partial; out & $\begin{array}{l}\text { weak closing } \\
\text { forces of the } \\
\text { clip and its } \\
\text { resterilisation. }\end{array}$ & $\begin{array}{c}\text { place several } \\
\text { clips or stack } \\
\text { one on the top } \\
\text { of another can } \\
\text { prevent clip } \\
\text { slippage. }\end{array}$ & postop DSA \\
\hline
\end{tabular}

Blood blister-like ICA

\begin{tabular}{|c|c|c|c|c|c|c|c|c|}
\hline 48 & Diraz [40] & 1993 & ICA (BBA) & unk & total; out & $\begin{array}{c}\text { due to brain } \\
\text { retraction } \\
\text { release }\end{array}$ & $\begin{array}{c}\text { Embedding the } \\
\text { clip by tearing } \\
\text { a small }\end{array}$ \\
\hline 49 & Park [41] & 2007 & ICA (BBA) & unk & total; out & unk & $\begin{array}{c}\text { postop } \\
\text { DSA } \\
\text { (5 wks) }\end{array}$ \\
\hline 50 & Kuroda [42] & 2016 & $\begin{array}{c}\text { ICA } \\
\text { (anterior } \\
\text { wall) }\end{array}$ & 1 bayonet & total; out & $\begin{array}{c}\text { radiation } \\
\text {-induced } \\
\text { severe }\end{array}$ & postop none \\
(5 wks)
\end{tabular}

BA

\begin{tabular}{|c|c|c|c|c|c|c|c|c|}
\hline 51 & Melo [35] & 2002 & BA & unk & total; out & & & $\begin{array}{c}\text { loss of } \\
\text { consciousness }\end{array}$ \\
\hline 52 & Miyachi [43] & 1999 & BA & unk & total; out & unk & none & postop DSA \\
\hline
\end{tabular}


Late postoperative slippage of the cerebral aneurysm clip...

63

\begin{tabular}{|c|c|c|c|c|c|c|c|c|}
\hline 54 & Drake [32] & 1984 & BA & 1 Sundt & total; out & unk & postop DSA & fatal rebleeding \\
\hline 56 & Drake [46] & 1996 & large BA & unk & partial; in & neck shape & none & $\begin{array}{l}\text { clip blades } \\
\text { stenosed the } \\
\text { origins of SCA; } \\
\text { ischemia }\end{array}$ \\
\hline 57 & Silverberg [47] & 1981 & giant BA & unk & unk & unk & $\begin{array}{c}\text { apart from } \\
\text { slippage, the } \\
\text { aneurysm has } \\
\text { thrombosed. }\end{array}$ & postop DSA \\
\hline
\end{tabular}

VA

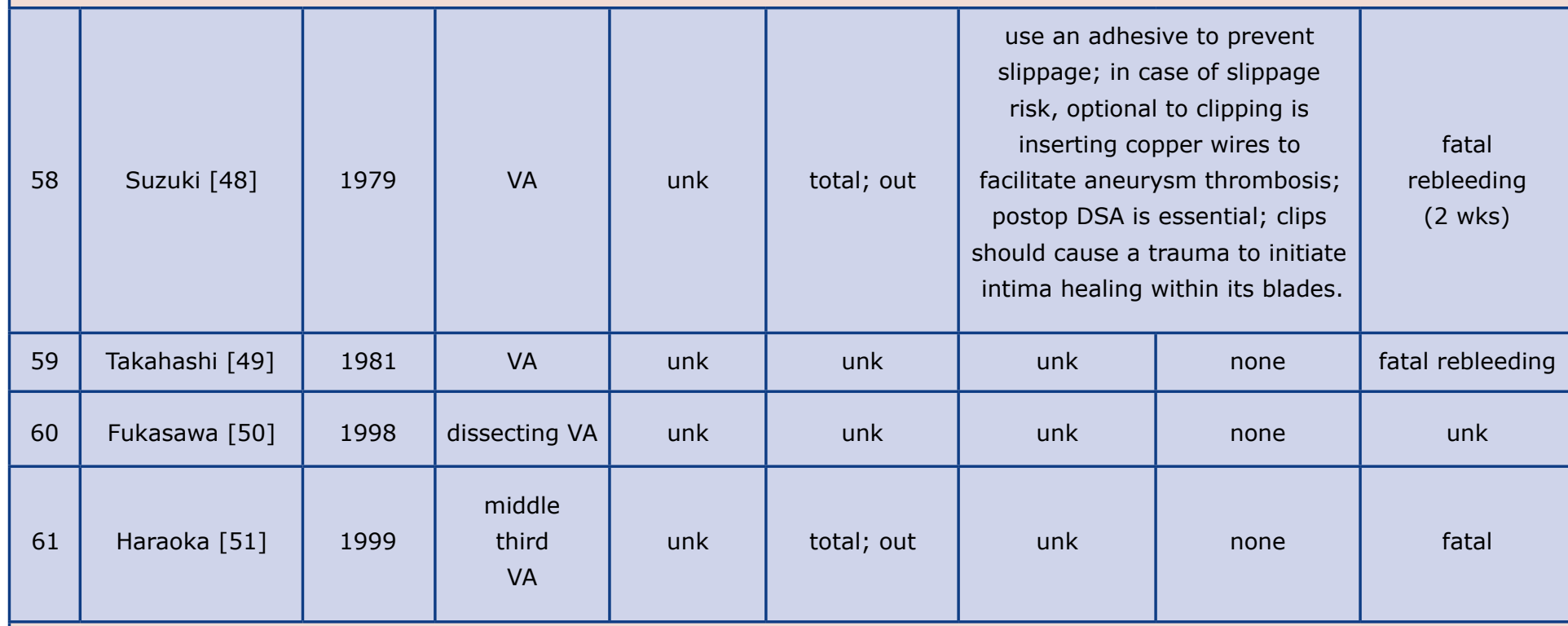

PICA

\begin{tabular}{|c|c|c|c|c|c|c|c|c|}
\hline 62 & Drake [46] & 1984 & PICA & older clip & total; out & unk & none & fatal rebleeding \\
\hline 63 & Oyesiku [52] & 1986 & PICA & Heifetz & total; out & \multicolumn{2}{|c|}{$\begin{array}{c}\text { The clip migrated to cauda } \\
\text { equine (L3-4). "Force of retur- } \\
\text { ning brain" has been suggested } \\
\text { as a factor of slippage. }\end{array}$} & $\begin{array}{l}\text { low back } \\
\text { pain with } \\
\text { radiculopathy }\end{array}$ \\
\hline 64 & Porchet [53] & 1995 & PICA & 1 unk & total; out & unk & none & rebleeding \\
\hline 65 & Kang [54] & 2004 & PICA & unk & unk & unk & $\begin{array}{l}\text { endovascular } \\
\text { embolization }\end{array}$ & $\begin{array}{c}\text { postop DSA } \\
5 \text { days postop }\end{array}$ \\
\hline 66 & Kim [55] & 2009 & PICA & $\begin{array}{c}3 \\
\text { Yasargil } \\
\text { (straight, } \\
\text { fenestrated, } \\
\text { angled) }\end{array}$ & total; out & \multicolumn{2}{|c|}{$\begin{array}{l}\text { The angled clip migrated } \\
\text { to sacral (S1) } \\
\text { subarachnoid space. } \\
\text { The reason of slippage } \\
\text { is unknown. }\end{array}$} & low back pain \\
\hline
\end{tabular}




\section{Other locations}

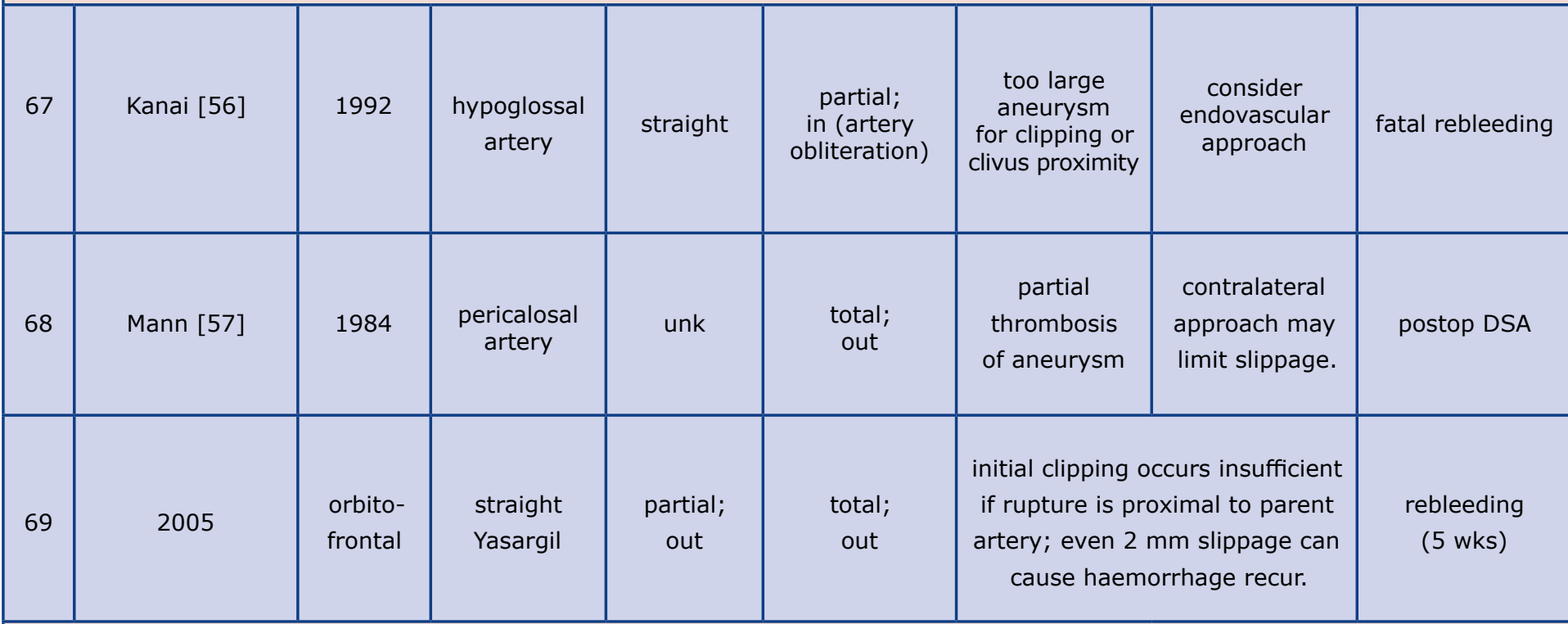

\section{Unspecified location}

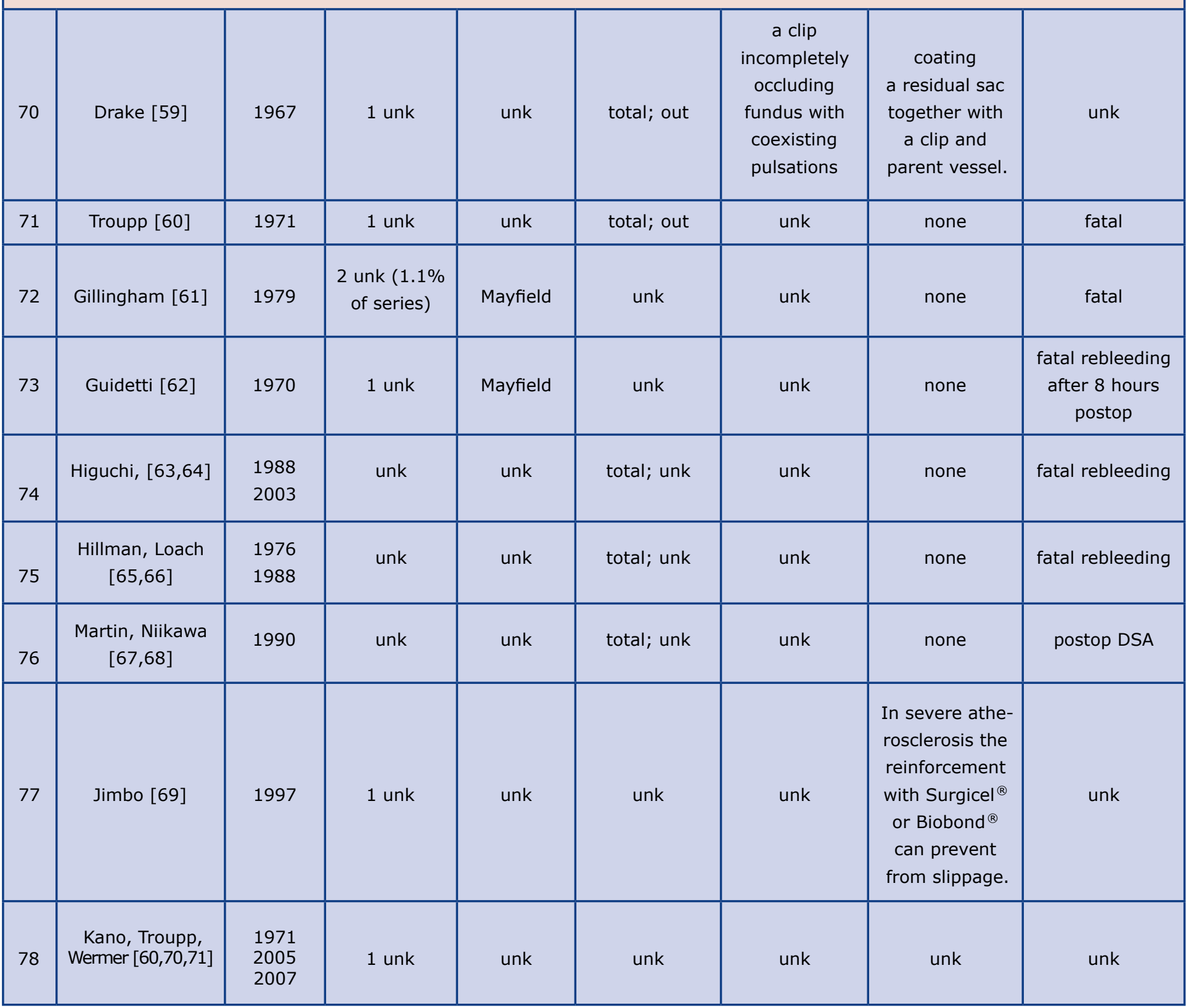




\begin{tabular}{|c|c|c|c|c|c|c|c|c|}
\hline 79 & Park [72] & 2014 & $\begin{array}{c}8 \text { unk } \\
\text { (4 atherosc- } \\
\text { lerotic, } \\
4 \text { non-athe- } \\
\text { rosclerotic) }\end{array}$ & unk & unk & $\begin{array}{l}\text { sliding of the } \\
\text { clip due to } \\
\text { atherosclerotic } \\
\text { neck }\end{array}$ & $\begin{array}{l}\text { use } \\
\text { multiple } \\
\text { clips }\end{array}$ & unk \\
\hline 80 & Nievas [73] & 2007 & 7 cases & unk & total; out & unk & none & postop DSA \\
\hline 81 & $\begin{array}{c}\text { Shephard } \\
\text { [74] }\end{array}$ & 1983 & $\begin{array}{c}4 \text { cases; unk } \\
\text { aneurysms }\end{array}$ & unk & unk & unk & none & fatal rebleeding \\
\hline 82 & Sugita, [75] & 1976 & unk & Heifetz & unk & unk & $\begin{array}{l}\text { broad-necked } \\
\text { aneurysms } \\
\text { should be } \\
\text { secured by } \\
\text { clips with more } \\
\text { than } 80 \text { gm clo- } \\
\text { sing pressure. }\end{array}$ & postop DSA \\
\hline 83 & Sundt [76] & 1982 & unk & Heifetz & unk & unk & none & unk \\
\hline
\end{tabular}

\section{Proposed management aimed for prevention of further clip slippage}

\begin{tabular}{|c|c|c|c|c|}
\hline 84 & Iwama $[77,78]$ & 2004 & large M1 & Dome puncture prevent slipping in or out of aneurysm clip \\
\hline 85 & Yasargil [79] & 1974 & distal ACA & Coagulation of the neck produces a smaller neck, then less chance of clip slipping. \\
\hline 86 & Ohno $[80,81]$ & $\begin{array}{l}1992 \\
1999\end{array}$ & ICA, ACA & Sugita straight booster clip was used for preventing a slip-out of the first clip. \\
\hline 87 & Sasaki [82] & 1991 & ICA & In giant aneurysms additional clips should be applied to prevent first clip slippage. \\
\hline 88 & Inci $[83]$ & 2015 & ICA & more long clips were placed parallel to the first clip on calcified-necked aneurysm \\
\hline 89 & $\begin{array}{l}\text { Hashimoto, } \\
\text { Kato }[84,85]\end{array}$ & $\begin{array}{l}1997 \\
2009\end{array}$ & ICA & $\begin{array}{l}\text { "interlocking" the tandem of angled fenestrated clip blades reinforce } \\
\text { their closing pressure and thus reduces the likelihood of slipping. }\end{array}$ \\
\hline 90 & Ohmoto $[86]$ & 1991 & $\begin{array}{l}\text { cavernous } \\
\text { ICA }\end{array}$ & reinforcing (booster) straight clip was used in wide-necked aneurysm \\
\hline 91 & Uemura [87] & 1987 & $\begin{array}{l}\text { paraclinoid } \\
\text { ICA }\end{array}$ & $\begin{array}{c}\text { For prevention of Sugita clip slipping, a small piece of dura is laid } \\
\text { between the spring and sphenoid with coating. }\end{array}$ \\
\hline 92 & Kataoka [88] & 1995 & $\begin{array}{l}\text { paraclinoid } \\
\text { ICA }\end{array}$ & cortex splitting to adjust a clip spring to prevent slippage. \\
\hline 93 & Gianotta $[89]$ & 1994 & ICA/Opth & $\begin{array}{l}\text { Clip slip off the aneurysm is frequent in ICA/Opth; } \\
\text { to avoid slippage series of clips should be stacked one on of top of another. }\end{array}$ \\
\hline 94 & Sengupta [90] & 1979 & $\begin{array}{c}\text { ICA } \\
\text { bifurcation }\end{array}$ & aneurysm sac was aspirated shortly after clipping to prevent further slippage \\
\hline
\end{tabular}




\begin{tabular}{|c|c|c|c|c|}
\hline 95 & $\begin{array}{l}\text { Fujioka, Shigeta } \\
{[91,92]}\end{array}$ & $\begin{array}{l}1992 \\
2003\end{array}$ & $\begin{array}{l}\text { ICA (BBA) or } \\
\text { dissecting }\end{array}$ & "clip on wrapping" method to prevent either intra- or postop slippage \\
\hline 96 & $\begin{array}{l}\text { Kato, Nakagawa, } \\
\text { Osawa [93-95] }\end{array}$ & $\begin{array}{l}1986 \\
1991 \\
1993\end{array}$ & ICA (BBA) & "Clipping on wrapping" with/without applied on cellulose fabric to prevent slippage. \\
\hline 97 & Kazumata [96] & 2014 & ICA (BBA) & Radial artery to MCA bypass graft is advocated decreases the risk of postop slippage. \\
\hline 98 & Yoshimoto [97] & 1996 & ICA (BBA) & wrapping with muslin gauze may prevent slipping. \\
\hline 99 & Otani $[98]$ & 2009 & ICA (BBA) & right-angled clip blades placed parallel to the parent artery prevent slippage \\
\hline 100 & Mooney [99] & 2015 & ICA (BBA) & placing a thin layer of cotton reinforcement beneath the clip blades \\
\hline 101 & Brown $[100]$ & 2017 & ICA (BBA) & clip blades should be placed along the axis of ICA \\
\hline 102 & Drake $[46]$ & 1996 & $\begin{array}{l}\text { BA } \\
\text { bifurcation }\end{array}$ & $\begin{array}{l}\text { In order to prevent further clip slipping down and stenosing/kinking the P1 } \\
\text { origins, a Drake proposed the tandem clipping, composed of one } \\
\text { fenestrated and one straight clip. }\end{array}$ \\
\hline 103 & Hirikoshi [101] & 1997 & $\begin{array}{l}\text { BA } \\
\text { bifurcation }\end{array}$ & $\begin{array}{l}\text { If clip blades slip toward BA closing the PCA origins, } \\
\text { direct clipping should be abandoned. }\end{array}$ \\
\hline 104 & Fujitsu [102] & 1994 & VA, BA & "wrap-clipping" technique with Dacron-meshed silastic sheet \\
\hline 105 & Sano $[103]$ & 1997 & $\begin{array}{l}\text { dissecting } \\
\text { VA }\end{array}$ & $\begin{array}{l}\text { a second curved fenestrated booster clip was placed on blades } \\
\text { of the first clip to eliminate its further slippage. }\end{array}$ \\
\hline 106 & Hylton [104] & 1988 & giant & atheroma removal from aneurysm sac should precede direct clipping \\
\hline 107 & Welch [105] & 1997 & giant & $\begin{array}{l}\text { intraaneurysmal thrombus prevents clips from closing and force the clip onto } \\
\text { the parent artery; partial thrombectomy while temporary clipping is advised. }\end{array}$ \\
\hline 108 & Wellman [106] & 1998 & giant & $\begin{array}{c}\text { clips placed across the neck require total occlusion, otherwise a pulsating } \\
\text { aneurysm neck pose a risk of slipping away or inwards. }\end{array}$ \\
\hline 109 & Kawai [107] & 1987 & giant & $\begin{array}{l}\text { To prevent slipping-in of the clip and artery occlusion, the dome thrombectomy, } \\
\text { neck thrombarterectomy, also using CUSA should follow neck clipping. }\end{array}$ \\
\hline 110 & $\begin{array}{l}\text { Lawton } \\
{[108,109]}\end{array}$ & $\begin{array}{l}1994 \\
1999\end{array}$ & giant & $\begin{array}{l}\text { intraaneurysmal thrombus prevents clips from closing and force the clip onto } \\
\text { the parent artery; partial thrombectomy while temporary clipping is advised. }\end{array}$ \\
\hline 111 & Symon [110] & 1992 & giant & $\begin{array}{l}\text { debulking the aneurysm and collapsing its neck diminish } \\
\text { the risk of clip slippage toward parent artery. }\end{array}$ \\
\hline 112 & Nakamura [111] & 2012 & wide-necked & multiple clipping to prevent clip slip-out. \\
\hline 113 & Nakano [112] & 2000 & wide-necked & "Clipping on wrapping" to prevent slip off. \\
\hline 114 & Turkmani [113] & 2015 & $\begin{array}{l}\text { aneurysms } \\
\text { with a calci- } \\
\text { fied neck }\end{array}$ & $\begin{array}{l}\text { a single clip can slip downward at the calcified neck } \\
\text { thus a clip reconstruction should be employed }\end{array}$ \\
\hline 115 & Kato [114] & 2012 & $\begin{array}{l}\text { previously } \\
\text { coiled }\end{array}$ & $\begin{array}{l}\text { Specific features of sac and neck of previously coiled aneurysm } \\
\text { should be considered preoperatively in order to avoid further slippage. }\end{array}$ \\
\hline 116 & Kiran [115] & 2015 & very small & double-clip technique (two parallel mini clips) prevents from slipping \\
\hline
\end{tabular}




\begin{tabular}{|c|c|c|c|c|}
\hline 117 & Giannotta [116] & 1995 & 4 unk & $\begin{array}{l}\text { Clip slippage was attributed to older style clips or their improper placement. } \\
\text { Recommended preventions: large portion of sac should be dissected first, } \\
\text { otherwise clip closing forces would not counteract tethering of fibrous material; } \\
\text { multiple and tandem clipping; use of booster clips; evacuating the sac; puncture the } \\
\text { sac once neck clipping is complete; do not place clips under hypotensive anaesthesia. }\end{array}$ \\
\hline 118 & Kato [117] & 1995 & unk & Fenestrated clip itself prevents slippage. \\
\hline 119 & Guo $[118]$ & 2007 & & excising a sac may contribute to a clip slippage \\
\hline 120 & Hollin [119] & 1973 & & persistence of blood pulsations to the clip \\
\hline 121 & $\begin{array}{c}\text { Hori, } \\
\text { Iwata, } \\
\text { Kato, } \\
\text { Kodama, Lee, } \\
\text { Mizoi, Sugita } \\
\text { [120-127] }\end{array}$ & $\begin{array}{l}1976 \\
1979 \\
1982 \\
1987 \\
1988 \\
1997\end{array}$ & & $\begin{array}{l}\text { additional wrapping/coating or adhesive } \\
\text { (i.e. cyanoacrylate) } \\
\text { use to prevent further slippage. }\end{array}$ \\
\hline 122 & Mayfield [128] & 1971 & Clip bla & should be parallel and incorporate as little of the surrounding tissue as possible. \\
\hline 123 & Nievas [129] & 2000 & $\begin{array}{l}\text { Develope } \\
\text { of sac fi } \\
\text { parallel to } \\
\text { instrume } \\
\text { tip, re } \\
\text { atheron } \\
\text { may }\end{array}$ & $\begin{array}{l}\text { veral tips to prevent clip slippage: use the mobile fulcrum clip, reduce the amount } \\
\text { (decrease blood pressure or use a temporary clip), place a second occluding clip } \\
\text { first one (then correct the first clip), never use a clip that has been left open in the } \\
\text { resterilized for a subsequent operation, leave a depth of at least } 2 \mathrm{~mm} \text { from the } \\
\text { completely the arachnoid bundles surrounding the aneurysm neck, remove the } \\
\text { efore a clip is applied on the ruptured ICA aneurysm, perpendicular clip insertion } \\
\text { to blades' cross, the neck resistance should be verified prior to clip placement. }\end{array}$ \\
\hline 124 & Nishi [130] & 2007 & $\begin{array}{l}\text { Wrap } \\
\text { slipping }\end{array}$ & $\begin{array}{l}\text { forced clipping for slippery aneurysm neck; sequential clip placement to avoid } \\
\text { nd occluding parent vessel (a pilot clip is removed after stabilizing a second clip). }\end{array}$ \\
\hline 125 & Nussbaum [131] & 2010 & & $\begin{array}{l}\text { dified fenestrated clip ("compression clip") was introduced to avoid slipping } \\
\text { from atheromatous, thrombotic or previously coiled aneurysms. }\end{array}$ \\
\hline 126 & Origitano [132] & 1997 & & Incture the sac and perform postop DSA to avoid slippage phenomena. \\
\hline 127 & Sano $[133]$ & 1991 & A doub & $\begin{array}{l}\text { cured aneurysm closure - fenestrated and straight clips closed across the neck; } \\
\text { that combination of clips initiated by Charles Drake. }\end{array}$ \\
\hline 128 & $\begin{array}{l}\text { Schmid } \\
\text {-Elsaesser [134] }\end{array}$ & 2000 & & broad-based aneurysms should be secured by more than one clip. \\
\hline 129 & Sughrue $[135]$ & 2011 & & orporating pathological tissues at the neck that can cause clip slippage \\
\hline 130 & Sugita $[136]$ & 1985 & $\begin{array}{l}\text { If a clip } \\
\text { Placin } \\
\text { puls }\end{array}$ & $\begin{array}{l}\text { onto the parent artery and causes stenosis, puncturing the sac is indispensable. } \\
\text { econd clip prevent slipping, even though the first clip do not open with arterial } \\
\text { n. Total wrapping after even successful clipping may prevent postop slipping. } \\
\text { Putting some chemical adhesives on clip blades. }\end{array}$ \\
\hline 131 & Sundt [137] & 1984 & & applying a booster clip prevent from slipping \\
\hline 132 & Safavi-Abbasi [138] & 2016 & & cotton-clipping and cotton-augmentation strategies \\
\hline 133 & Sakata [139] & 2015 & & clip and wrap technique using Gore-Tex sling \\
\hline
\end{tabular}




\section{Discussion}

Our systematic review was divided into two stages: we pooled all valid cases in which an aneurysm clip slid off and collected all studies addressing prevention of clip slippage. By including every type of study into the systematic review, we intended to reveal case reports and authors' own experiences. However, most authors $(65.3 \%)$ did not provide any reason why the clip slipped off. The incomplete clipping and insufficient amount of used clips were the most commonly stated reasons. On the other hand, tandem clipping seems more durable option proposed by $15.8 \%$ of authors in our systematic review [9, 18-20].

The prevention of clip slippage depended on aneurysm location. Specifically, reinforcing with any wrapping material, clip-wrapping methods and placing blades parallel to carotid were proposed in blood blister-like aneurysms [21-25]. Whereas in cases of a clip slipped from ACoA aneurysm, the authors did not provide any suggestions for repair.
Our systematic review pooled reports of slipped cerebral aneurysm clips. Based on this cohort we concluded that by using a single clip the surgeon should consider aneurysm recurrence. It was often speculated in the literature that multiple clipping more seems to be a more durable solution. Plenty of valuable hints on how to avoid postoperative clip slippage were suggested in the literature and we listed all of them based on the specific aneurysm location.

\section{Ethical approval}

Formal consent is not required for this type of study.

\section{Informed consent}

Informed consent was obtained from all individual participants included in the study.

\section{References}

1. Drake CG, Peerless SJ, Hernesniemi JA. Surgery of Vertebrobasilar Aneurysms [Internet]. Vienna: Springer Vienna; 1996. Available from: http://link.springer.com/10.1007/978-3-7091-9409-6

2. Giannotta SL. Complication A voidance for Large and Giant Carotid Ophthalmic Aneurysms. In. New Trends in Management of Cerebro-Vascular Malformations [Internet]. Pasqualin A, Da Pian R, editors. Vienna: Springer Vienna; 1994 [cited 2014 Sep 12]. 198-202 p. Available from: http://link.springer.com/10.1007/978-3-7091-9330-3

3. Szmuda T, Słoniewski P. Giant Intracranial Aneurysms - Surgical Treatment, Accessory Techniques and Outcome. In: Murai Y, editor. Aneurysm [Internet]. InTech; 2012 [cited 2014 Sep 5]. p. 351-82. Available from: http://cdn.intechopen. com/pdfs-wm/38617.pdf

4. Carvi y Nievas M, Höllerhage H. Risk of intraoperative aneurysm clip slippage: a new experience with titanium clips. J Neurosurg [Internet]. 2000 Mar [cited 2014 Sep 5];92(3):478-80. Available from: http://thejns.org/doi/abs/10.3171/ ins.2000.92.3.0478

5. Carvi y Nievas M. Assessment of the clipping efficacy of intracranial aneurysms: analysis of the employed methodology in relation to case difficulty. Neurol Res [Internet]. 2007 Jul [cited 2014 Sep 3];29(5):506-16. Available from: http:// www.ncbi.nlm.nih.gov/pubmed/17535574

6. Horiuchi T, Li Y, Seguchi T, Sato A, Aoyama T, Hanaoka Y, et al. Clip blade scissoring with titanium bayonet clip in aneurysm surgery. Two case reports. Neurol Med Chir (Tokyo) [Internet]. 2012 [cited 2017 Nov 26];52(2):84-6. Available from: http://www.ncbi.nlm.nih.gov/pubmed/22362289

7. Kariyattil R, Panikar D. Scissoring of a Cobalt Alloy Aneurysm Clip causing Slippage during Cerebral Aneurysm Surgery: Case report and review of literature. Sultan Qaboos Univ Med J [Internet]. 2013 Feb [cited 2014 Sep 2];13(1):17982. Available from: http://www.pubmedcentral.nih.gov/articlerender.fcgi?artid=3616789\&tool=pmcentrez\&rendertype=abstract

8. Asgari S, Wanke I, Schoch B, Stolke D. Recurrent hemorrhage after initially complete occlusion of intracranial aneurysms. Neurosurg Rev [Internet]. 2003 Oct [cited 2014 Sep 2];26(4):269-74. Available from: http://www.ncbi.nlm.nih. gov/pubmed/12802695

9. Giannotta SL, Litofsky NS. Reoperative management of intracranial aneurysms. J Neurosurg [Internet]. 1995 Sep [cited 2014 Sep 2];83(3):387-93. Available from: http://www.ncbi.nlm.nih.gov/pubmed/7666212

10. McKissock W. Recurrence of an Intracranial Aneurysm after Excision. J Neurosurg [Internet]. 1965 Nov [cited 2017 Nov 26];23(5):547-8. Available from: http://www.ncbi.nlm.nih.gov/pubmed/5858447 
11. Kim YH, Kim JE, Kang H-S, Han DH. Migration of an aneurysm clip to the sacral subarachnoid space. Acta Neurochir (Wien) [Internet]. 2009 Mar 10 [cited 2014 Sep 2];151(6):699-700. Available from: http://www.scopus.com/inward/ record.url?eid=2-s2.0-67650444427\&partnerID=tZOtx3y1

12. Oyesiku NM, Jones RK. Migration of a Heifetz aneurysm clip to the cauda equina causing lumbar radiculopathy. Case report. J Neurosurg [Internet]. 1986 Aug [cited 2014 Sep 5];65(2):256-7. Available from: http://www.ncbi.nlm.nih.gov/ pubmed/2941527

13. Hayashi Y, Kimura M, Satake R, Kinoshita A. Possible participation of clip rotation in the formation of de novo aneurysm. J Clin Neurosci [Internet]. 2004 Apr [cited 2014 Sep 6];11(3):331-4. Available from: http://www.sciencedirect.com/ science/article/pii/S0967586803001991

14. Beltagy M El, Muroi C, Roth P, Fandino J, El Beltagy M, Imhof H-G, et al. Recurrent Intracranial Aneurysms After Successful Neck Clipping. World Neurosurg [Internet]. 2010 [cited 2014 Sep 5];74:472-7. Available from: http://www. sciencedirect.com/science/article/pii/S1878875010002846

15. Szmuda T, Słoniewski P. Postoperative cerebral aneurysm clip slippage: systematic review. PROSPERO 2014 CRD42014013843 [Internet]. Available from: https://www.crd.york.ac.uk/prospero/display record.php?RecordID $=13843$

16. Moher D, Liberati A, Tetzlaff J, Altman DG. Preferred reporting items for systematic reviews and meta-analyses: the PRISMA statement. PLoS Med [Internet]. 2009 Jul 21 [cited 2014 Mar 20];6(7):e1000097. Available from: http://www. pubmedcentral.nih.gov/articlerender.fcgi?artid=2707599\&tool=pmcentrez\&rendertype=abstract

17. Stewart LA, Clarke M, Rovers M, Riley RD, Simmonds M, Stewart G, et al. Preferred Reporting Items for Systematic Review and Meta-Analyses of individual participant data: the PRISMA-IPD Statement. JAMA [Internet]. 2015 Apr 28 [cited 2016 Dec 27];313(16):1657-65. Available from: http://iama.jamanetwork.com/article.aspx?doi=10.1001/ jama.2015.3656

18. Wester K. Lessons learned by personal failures in aneurysm surgery: what went wrong, and why? Acta Neurochir (Wien) [Internet]. 2009 Sep [cited 2014 Sep 3];151(9):1013-24. Available from: http://www.scopus.com/inward/record.url?eid=2-s2.0-69249213985\&partnerID=tZOtx3y1

19. Park CK, Shin HS, Choi SK, Lee SH, Koh JS, Martin N. Clinical Analysis and Surgical Considerations of Atherosclerotic Cerebral Aneurysms: Experience of a Single Center. J Cerebrovasc Endovasc Neurosurg [Internet]. 2014 Sep 1 [cited 2017 Jul 10];16(3):247. Available from: https://synapse.koreamed.org/DOlx.php?id=10.7461/icen.2014.16.3.247

20. Nakamura K, Kitabayashi M, Murata T. Clipping for Wide-necked Asymptomatic Unruptured Intracranial Aneurysm. Surg Cereb Stroke [Internet]. 2012 Mar 19 [cited 2014 Sep 7];40(4):251-6. Available from: https://www.jstage.jst.go.jp/ article/scs/40/4/40 251/ article

21. Fujioka S. clipping on wrapping method for treatment of short aneurysms. Surg Cereb Stroke [Internet]. 2003 [cited 2014 Sep 4];31:375-9. Available from: $\underline{\text { http://ilc.jst.go.jp/JST.JSTAGE/scs/31.375?from=Google }}$

22. Shigeta H, Kyoshima K, Nakagawa F, Kobayashi S. Dorsal internal carotid artery aneurysms with special reference to angiographic presentation and surgical management. Acta Neurochir (Wien). 1992;119(1-4):42-8.

23. Yoshimoto Y, Ochiai C, Nagai M. Cerebral aneurysms unrelated to arterial bifurcations. Acta Neurochir (Wien). 1996;138(8):958-63.

24. Mooney MA, Kalani MYS, Nakaji P, Albuquerque FC, McDougall CG, Spetzler RF, et al. Long-term Patient Outcomes After Microsurgical Treatment of Blister-Like Aneurysms of the Basilar Artery. Neurosurgery [Internet]. 2015 Sep [cited 2016 Dec 27];11 Suppl 3:387-93. Available from: http://content.wkhealth.com/linkback/openurl?sid=WKPTLP:landingpage \&an=00006123-201509001-00005

25. Brown MA, Guandique CF, Parish J, McMillan AC, Lehnert S, Mansour N, et al. Long-term follow-up analysis of microsurgical clip ligation and endovascular coil embolization for dorsal wall blister aneurysms of the internal carotid artery. J Clin Neurosci [Internet]. 2017 May [cited 2017 Nov 26];39:72-7. Available from: http://linkinghub.elsevier.com/retrieve/pii/S0967586816309419 